\title{
The hydrino and other unlikely states
}

\author{
Norman Dombey ${ }^{a}$ \\ Department of Physics and Astronomy \\ University of Sussex \\ Brighton BN1 9QH, UK \\ ${ }^{a}$ normand@sussex.ac.uk,
}

We discuss the tightly bound (hydrino) solution of theKlein-Gordon equation for the Coulomb potential in 3 dimensions. We show that a similar tightly bound state occurs for the Dirac equation for the Coulomb potential in 2 dimensions. These states are unphysical since they disappear if the nuclear charge distribution is taken to have an arbitrarily small but non-zero radius.

(August 8, 2006)

\section{THE HYDRINO STATE}

Naudts [1] recently drew attention to the so-called hydrino state of hydrogen, a strongly bound state which has a normalisable solution of the Klein-Gordon equation for a Coulomb potential in three space dimensions, but no analogue in the Schrödinger equation. It has been mentioned by a minority of quantum mechanics textbooks in the past [2], which discard it for various ad hoc reasons: for instance, because it lacks a nonrelativistic analogue, or because it yields divergent expressions for the expectation value of the potential energy. Claims that a tightly-bound state of hydrogen exists have, however, been widely publicised [3]. Another relativistic equation, the Bethe-Salpeter equation, has been shown to have anomalous solutions which on closer inspection are unphysical [4].

In this letter, we show that similar anomalous tightlybound states for a Coulomb potential also exist in the Dirac equation in two space dimensions. We claim that all these anomalous states are unphysical by developing an argument used by Schiff [5], which was first used by Loudon [6] for the one dimensional Schrödinger equation with the potential $-\alpha /|x|$.

We shall begin with a brief discussion of the hydrino state for the Coulomb potential $V(r)=-\alpha / r$ which is taken to be the fourth component of a four-vector. If the solution $\psi(r, \theta, \varphi)$ for the Klein-Gordon equation of a particle of mass $m$ is separated out into its radial and angular components in the usual way and we consider only states of angular momentum $l=0$, then if $f(r)=r \psi$ we have (in natural units where $\hbar=1=c$ )

$$
\frac{d^{2} f}{d r^{2}}+\left[\left(E+\frac{\alpha}{r}\right)^{2}-m^{2}\right] f(r)=0
$$

Consider bound states so that energy $E<m$. Write $\kappa=\sqrt{m^{2}-E^{2}}, z=2 \kappa r$ and then

$$
f^{\prime \prime}(z)+\left[-\frac{1}{4}+\frac{\lambda}{z}+\frac{\left(1 / 4-\mu^{2}\right)}{z^{2}}\right] f(z)=0
$$

where $\lambda=\alpha E / \kappa$ and $\mu=+\sqrt{1 / 4-\alpha^{2}}$. Eq. (2) is called Whittaker's equation [7] and the solutions can be expressed in terms of confluent hypergeometric functions. To see this take

$$
f(z)=z^{\mu+1 / 2} e^{-z / 2} w(z)
$$

to obtain the defining confluent hypergeometric or Kummer's equation [7]

$$
z w^{\prime \prime}+(b-z) w^{\prime}-a w=0 ; a=\mu+1 / 2-\lambda, b=2 \mu+1
$$

The roots $t$ of the indicial equation, giving the lowest power of $z$ when $w$ is expressed as a power series, are $t=0$ and $t=1-b=-2 \mu$. The solutions of Eq.(4) which allow the wave function $\psi$ to be square integrable to infinity are polynomials $w(z)=M(a, b, z)$ with $a=-N$ where $N$ is a non-negative integer.

The conventional choice is $t=0$; then $\lambda_{N}=\mu+1 / 2+$ $N$, whence

$$
E_{N} / m=\left[1+\frac{\alpha^{2}}{\left(N+1 / 2+\sqrt{1 / 4-\alpha^{2}}\right)^{2}}\right]^{-1 / 2}
$$

In particular the ground-state energy $E_{0}$ is given by

$$
E_{0} / m=(1 / \sqrt{2}) \sqrt{1+\sqrt{1-4 \alpha^{2}}}
$$

It is easy to show that to lowest order in small $\alpha$ Eq.(5) gives the non-relativistic spectrum for the hydrogen atom obtained from the Schrödinger equation.

There are also anomalous solutions of Eq. (2) from the other root $t=-2 \mu$ (or equivalently set $\mu=$ $\left.-\sqrt{1 / 4-\alpha^{2}}\right)$. They give the spectrum

$$
E_{N A} / m=\left[1+\frac{\alpha^{2}}{\left(N+1 / 2-\sqrt{1 / 4-\alpha^{2}}\right)^{2}}\right]^{-1 / 2}
$$

and in particular

$$
E_{0 A} / m=(1 / \sqrt{2}) \sqrt{1-\sqrt{1-4 \alpha^{2}}}
$$


This is the solution which is called the hydrino. It is normalisable but has no counterpart in the Schrodinger equation. Indeed for small $\alpha, E_{0 A}=m \alpha$ and the electron is tightly bound.

It is tempting to say with Migdal [8] that the one particle Klein-Gordon equation cannot be taken seriously and field theory is required. But we now demonstrate similar tightly bound states for small $\alpha$ in the one-particle Dirac equation, where tight binding should not cause a problem [9].

\section{THE COULOMB POTENTIAL IN THE TWO-DIMENSIONAL DIRAC EQUATION}

This problem has been discussed previously [10] and anomalous solutions were noticed by Sigurdsson [11]. In two space-dimensions we can take the gamma matrices $\gamma_{x}, \gamma_{y}$ and $\gamma_{0}$ to be the Pauli matrices $i \sigma_{x}, i \sigma_{y}$ and $\sigma_{z}$ respectively. Then the Dirac equation for a particle of definite energy $E$ in the presence of the potential $V(r)=$ $-\alpha / r$ is

$$
\left[\sigma_{x} \frac{\partial}{\partial x}+\sigma_{y} \frac{\partial}{\partial y}-(E-V) \sigma_{z}+m\right] \psi=0
$$

Convert to polar coordinates and take

$$
\psi(r, \theta)=\left(\begin{array}{c}
f(r) e^{i(j-1 / 2) \theta} \\
g(r)^{i(j+1 / 2) \theta}
\end{array}\right)
$$

which is an eigenstate of the z-component of total angular momentum $J_{z}=L_{z}+\frac{1}{2} \sigma_{z}$ with eigenvalue $j$. Eq. (8) now becomes

$$
\begin{array}{r}
(m-E-\alpha / r) f+d g / d r+(j+1 / 2) g / r=0 \\
d f / d r-(j-1 / 2) f / r+(E+\alpha / r+m) g=0
\end{array}
$$

We follow the approach of Tutik [12]. Write

$$
f=F \sqrt{(1+E / m)}, \quad g=G \sqrt{(1-E / m)}
$$

and $\Phi_{1,2}=F \pm G$, and scale to $z$ to obtain

$$
\begin{aligned}
& \frac{d \Phi_{1}}{d z}+\frac{\Phi_{1}}{2 z}-j \frac{\Phi_{2}}{z}+\frac{1}{2} \Phi_{1}=\frac{\alpha}{\kappa z}\left(E \Phi_{1}+m \Phi_{2}\right) \\
& \frac{d \Phi_{2}}{d z}+\frac{\Phi_{2}}{2 z}-j \frac{\Phi_{1}}{z}-\frac{1}{2} \Phi_{2}=-\frac{\alpha}{\kappa z}\left(m \Phi_{1}+E \Phi_{2}\right)
\end{aligned}
$$

Take $\Phi_{1,2}=\phi_{1,2} \exp (-z / 2) ;$ then

$$
z \frac{d \phi_{1}}{d z}+\left(\frac{1}{2}-\frac{\alpha E}{\kappa}\right) \phi_{1}=\left(j+\frac{\alpha m}{\kappa}\right) \phi_{2}
$$

so $\phi_{2}$ can be written in terms of $\phi_{1}$. The second order differential equation for $\phi_{1}$ is

$$
\begin{aligned}
& z \frac{d}{d z}\left[z \frac{d \phi_{1}}{d z}+\left(\frac{1}{2}-\frac{\alpha E}{\kappa}\right) \phi_{1}\right]+ \\
& \left(\frac{\alpha E}{\kappa}+\frac{1}{2}-z\right)\left[z \frac{d \phi_{1}}{d z}+\left(\frac{1}{2}-\frac{\alpha E}{\kappa}\right) \phi_{1}\right] \\
= & \left(j^{2}-\frac{\alpha^{2} m^{2}}{\kappa^{2}}\right) \phi_{1}
\end{aligned}
$$

or

$$
\begin{aligned}
& z \phi_{1}^{\prime \prime}(z)+(2-z) \phi_{1}^{\prime}-(1 / 2-\alpha E / \kappa) \phi_{1} \\
= & \left(j^{2}-1 / 4-\alpha^{2}\right) \phi_{1} / z
\end{aligned}
$$

Write $\phi_{1}=z^{t} w$ so we obtain

$$
\begin{aligned}
& z w^{\prime \prime}(z)+(2+2 t-z) w^{\prime}+\left(t^{2}+t\right) w / z-t w \\
= & \left(\frac{j^{2}-1 / 4-\alpha^{2}}{z}+\frac{1}{2}-\frac{\alpha E}{\kappa}\right) w
\end{aligned}
$$

Hence take

$$
t^{2}+t=j^{2}-1 / 4-\alpha^{2}
$$

or

$$
t=-1 / 2 \pm \sqrt{j^{2}-\alpha^{2}}
$$

to obtain

$$
z w^{\prime \prime}(z)+(2+2 t-z) w^{\prime}-\left(t+\frac{1}{2}-\frac{\alpha E}{\kappa}\right) w=0
$$

which again is Kummer's equation. The regular solution $M(a, b, z)$ is square integrable to infinity only if it reduces to a polynomial, which it does if

$$
a=t+1 / 2-\alpha E / \kappa=-N
$$

where $N$ is a non-negative integer.

Conventionally one chooses the positive sign in Eq. (17) which yields

$$
\alpha E_{N} / \kappa=N+\sqrt{j^{2}-\alpha^{2}}
$$

or for $j=\frac{1}{2}$

$$
\frac{E_{N}}{m}=\left[1+\frac{\alpha^{2}}{\left(N+\sqrt{1 / 4-\alpha^{2}}\right)^{2}}\right]^{-1 / 2}
$$

and the ground state is given by

$$
E_{0} / m=\sqrt{1-4 \alpha^{2}}
$$

As $r \rightarrow 0, f, g \sim r^{t}=r^{-\frac{1}{2}+\sqrt{\frac{1}{4}-\alpha^{2}}}$ so that the wave functions remain square-integrable down to the origin even though they diverge. They are the relativistic generalisation of the solutions of the Schrödinger equation in two dimensions which has the energy spectrum given by Eq. (21) to lowest order in $\alpha^{2}$. 
The anomalous solutions arise from the other root of Eq. (17): $t=-1 / 2-\sqrt{1 / 4-\alpha^{2}}$. They too are square integrable down to the origin. The anomalous energy eigenvalues are given by $a=t+1 / 2-\alpha E_{A} / \kappa=-N$ or

$$
\alpha E_{N A} / \kappa=N-\sqrt{1 / 4-\alpha^{2}}
$$

Because $\kappa>0$, the lowest anomalous state with $N=0$ satisfies

$$
\alpha E_{0 A} / \kappa=-\sqrt{1 / 4-\alpha^{2}}
$$

whence

$$
E_{0 A} / m=-\sqrt{1-4 \alpha^{2}}
$$

This solution has no non-relativistic analogue and like the hydrino corresponds to a state which is tightly bound for small $\alpha$.

\section{DISCUSSION}

We believe that the three dimensional Klein-Gordon hydrino state and its two dimensional Dirac analogue are unphysical in spite of having normalisable wave functions. Their obvious failings are that

1. they lack non-relativistic counterparts even for arbitrarily small coupling;

2. the states persist even when the coupling is turned off;

3. the strength of the binding increases as the coupling strength $\alpha$ decreases. The maximum binding occurs for $\alpha=0$ when the potential has disappeared completely ${ }^{1}$.

These reasons may be sound but they are not decisive either mathematically or physically. We now demonstrate that, if the point charge of the nucleus is replaced by a charge extending over an arbitrarily small but finite radius $R$, then the anomalous functions become unacceptable because for small enough $R$ they cease to satisfy the appropriate wave equation. We will prove this for the Klein-Gordon equation; the argument for the Dirac case runs similarly.

For simplicity, we look at a conducting spherical shell of radius $r=R$, so that $V(r<R)=-\alpha / R$ while $V(r>$ $R)=-\alpha / r$. We need consider only $\alpha^{2} \ll 1$ since we are investigating the bound-state energy in the limit $\alpha \rightarrow 0$. Then the inner solution of Eq. (1) is

$$
f(r<R)=C \sin (q r), \quad q=\sqrt{(E+\alpha / R)^{2}-m^{2}}
$$

\footnotetext{
${ }^{1}$ We could call these anomalous states homeopathic states because the smaller the coupling, the larger the effect.
}

For $R$ small enough to make $\alpha / R>>m$, one has $\quad q \rightarrow$ $\alpha / R$, so that $q r=\alpha r / R \leq \alpha \ll 1 \Rightarrow f(r)=\operatorname{Cqr}(1+$ $O\left((q r)^{2}\right)$. In terms of the scaled variable $z$, and defining $Z \equiv 2 \kappa R$, this entails

$$
\left.\frac{z d f(z) / d z}{f(z)}\right|_{z=Z}=1+O(Z)
$$

This solution has to be joined to the outer solution of Eq. (2) for $z>Z=2 \kappa R$. For $w(z)$ defined by (??) the general solution of Eq. (4) reads [7]

$$
w=A M(a, b, z)+B z^{1-b} M(a-b+1,2-b, z)
$$

where as $z \rightarrow \infty$

$$
M(c, d, z \rightarrow \infty) \rightarrow \frac{\Gamma(d)}{\Gamma(c)} \exp (z) z^{c-d}
$$

Hence

$$
w(z \rightarrow \infty) \rightarrow\left\{A \frac{\Gamma(b)}{\Gamma(a)}+B \frac{\Gamma(2-b)}{\Gamma(a-b+1)}\right\} \exp (z) z^{a-b} .
$$

For a bound state the expression in the brackets must vanish so that

$$
\begin{aligned}
w= & M(a, b, z)- \\
& \frac{\Gamma(b) \Gamma(a-b+1)}{\Gamma(a) \Gamma(2-b)} z^{1-b} M(a-b+1,2-b, z)
\end{aligned}
$$

Thus for small $z$

$$
\begin{aligned}
& w(z \rightarrow 0) \rightarrow 1- \\
& \quad \frac{\Gamma(b) \Gamma(a-b+1)}{\Gamma(a) \Gamma(2-b)}\left\{z^{1-b}+\frac{(a-b+1)}{(2-b)} z^{2-b}+\ldots\right\}
\end{aligned}
$$

hence

$$
f(z \rightarrow 0) \rightarrow(1-z / 2+\ldots) z^{\mu+1 / 2} w
$$

Recall $a=\mu+1 / 2-\alpha E / \kappa, b=2 \mu+1, \mu=\sqrt{1 / 4-\alpha^{2}}$, so for small $\alpha$

$$
1-b \simeq-1+2 \alpha^{2}, \quad 2-b \simeq 2 \alpha^{2} .
$$

Since we are considering the limit as $\alpha \rightarrow 0$, we can disregard $\alpha^{2}$ in the various exponents of $z$ and so as $z \rightarrow 0$

$$
f=z-\frac{\Gamma(b) \Gamma(a-b+1)}{\Gamma(a) \Gamma(2-b)}(1+z+\ldots)
$$

Hence requiring $f(z)$ to be proportional to $z$ for small $z$ in order to satisfy Eq. (26)gives

$$
1 / \Gamma(a)=0 \quad \Rightarrow \quad a=-N, \quad N=0,1,2, \ldots
$$

This is precisely the conventional quantisation condition which gives the energy eigenvalue spectrum of Eq. (5) thus eliminating the anomalous solutions.

For the two dimensional Dirac equation for a Coulomb potential a similar argument, with Bessel functions replacing trigonometric functions for the inner solution, will give $f(r=R) \rightarrow$ const; $g(r=R) \rightarrow 0$ for $R$ small enough and hence $\phi_{1}=z^{t} w \rightarrow$ const. This is only possible if the positive sign is taken in Eq. (17), ruling out the anomalous solutions here too. 


\section{CONCLUSIONS}

We have shown that there are normalisable solutions of the Dirac equation for the Coulomb potential in two dimensions which are tightly bound, just like the hydrino solution of the Klein-Gordon equation in three dimensions. We have displayed the unphysical nature of such solutions by smearing the nuclear charge distribution so that it has an arbitrarily small non-zero radius; in that case there are no anomalous solutions. We suggest that outside of science fiction this is sufficient reason to disregard them. We leave to others to find a general criterion to exclude the anomalous solutions for the Coulomb potential with a point charge in two and three space dimensions: in the Appendix we indicate a possible route to this end.

Acknowledgements I would like to thank Gabriel Barton for suggesting that the Coulomb potential should be cut off at small distances and showing me how to manipulate the confluent hypergeometric functions. I would also like to thank the referee for suggesting the insertion of the Appendix.

\section{APPENDIX}

The requirement that wave functions have to be square integrable is not the only restriction on physical states in quantum mechanics. As de Castro has pointed out in his study of Klein Gordon particles [13] for a similar problem, it is also necessary to ensure that the Hamiltonian is Hermitian. We shall not attempt to do this here, but a necessary consequence of Hermiticity will be that the eigenfunctions of the Hamiltonian corresponding to two distinct energy eigenvalues are orthogonal.

The eigenfunctions $f_{0}(z)$ for the normal ground state energy given by Eq. (6) and $f_{0 A}(z)$ for the hydrino energy given by Eq. (7) are similar. They are both of the form given by Eq. (3):

$$
f(z)=z^{\mu+1 / 2} e^{-z / 2} w(z)
$$

where in both cases $w(z)=M(a, b, z)$ and $a=0$; i.e. $w(z)$ is constant. Neither $f_{0}(z)$ nor $f_{0 A}(z)$ vanishes except at $z=0$. Hence the hydrino wave function $\psi_{0 A}=f_{0 A}(z) / r$ cannot be orthogonal to $\psi_{0}=f_{0}(z) / r$. Thus the hydrino state cannot be physical provided that it is possible to ensure that the Hamiltonian for the hydrogen atom in the Klein-Gordon equation is Hermitian.

[1] J. Naudts On the Hydrino State of the Relativistic Hydrogen Atom, arxiv.physics/0507193 v2 (2005)
[2] L.I. Schiff Quantum mechanics, 2nd ed. McGraw-Hill, New York (1955); A.S.Davydov Quantum mechanics, Pergamon Press, Oxford (1965); H.A.Bethe and R.W.Jackiw, Intermediate Quantum Mechanics, 3rd ed. Benjamin, Menlo Park (1988)

[3] T. Reichhardt, Nature 42011 (2002); A. Jha, The Guardian, November 4 2005; R. Matthews, Financial Times, March 102006

[4] R.E.Cutkosky, Phys Rev. 961135 (1954); N.Nakanishi, Phys. Rev. 138B 1182 (1965)

[5] L.I. Schiff L I Quantum mechanics, 3rd ed, McGraw-Hill, Singapore (1968); Section 51, problem 3.

[6] R. Loudon, Am. J. Phys. 27649 (1959)

[7] M. Abramowitz and I.A.Stegun, Handbook of Mathematical Functions, Dover, New York (1965)

[8] A.B.Migdal, Rev. Mod. Phys. 50116 (1978)

[9] A. Calogeracos and N. Dombey, Phys. Rev. Lett. 92 040406 (2004)

[10] S.A.Bruce, Am J Phys 54446 (1986); S-H Dong and Z-Q Ma, Phys. Lett. A312 76 (2003)

[11] S. Sigurdsson, University of Sussex undergraduate project (1985) (unpublished)

[12] R.S.Tutik, J. Phys. A 25 L413 (1992)

[13] A.S. de Castro, Phys. Lett. A338 81 (2005) 